\title{
Evaluation of Doppler-guided hemorrhoidal artery ligation in the management of symptomatic hemorrhoids
}

\author{
Reda S Mohammed, MD; Hamed H Abo Steit, MD, MRCS; \\ Ahmed M Kamal, MD; Mostafa F Abdellateif, MD
}

\author{
Department of General Surgery, Ain Shams University, Cairo, Egypt.
}

\begin{abstract}
Background: We evaluated the results of the Doppler-guided hemorrhoidal artery ligation procedure in the management of symptomatic hemorrhoids to determine its effectiveness, safety, short and long term outcome.

Patients and methods: Between January 2007 and December 2008, fifty consecutive patients with symptomatic hemorrhoids were treated by Doppler-guided hemorrhoidal artery ligation method. All patients were treated in insurance hospital in Riyad, KSA and Palestinian hospital, Cairo, Egypt. Postoperative pain was measured with visual analog scale (1-10). Patients were followed up at intervals of one week, 1, 3, 6 and 12 months postoperatively. At every postoperative follow up visit, wound healing was observed and any complication was reported.

Results: Thirty five patients had their operations performed under general anesthesia. The operative time ranged between 19-45 minutes (mean $32 \pm 3.6$ minutes). The mean postoperative pain score was $6.1 \pm 1.3$ in the first postoperative day. The complications rate was low and the most common long term complication was persistent prolapse in three patients (6\%).

Conclusion: Doppler-guided hemorrhoidal artery ligation procedure in the management of symptomatic hemorrhoids is safe, effective and is associated with minimal long term complications. The procedure can be done on an out-patient basis.
\end{abstract}

\section{Introduction:}

Hemorrhoids is a common disease that has been reported to affect $8-10 \%$ of the general population. ${ }^{1}$ The choice of treatment modality depends on the degree of hemorrhoids and the local experience. ${ }^{2,3}$ Generally speaking, early hemorrhoids are frequently treated conservatively by fiber-rich diet, suppositories and better defecation discipline.1,4 More advanced disease needs surgery that is frequently associated with severe postoperative pain. It is the pain after hemorroidectomy that makes many patients refuse surgery. A lot of procedures have been tried to decrease postoperative pain after hemorrhoidectomy such as the addition of lateral sphincterotomy, anal dilatation, and local anesthetic injection ${ }^{3,5}$ and more recently stapled hemorrhoidopexy. ${ }^{6}$

Doppler-guided hemorrhoidal artery ligation (DG-HAL) is a new method for treatment of symptomatic hemorrhoids with minimal postoperative pain. It was first described in
1995. It is based on the theory that hemorrhoids occur when there is an imbalance in the blood flow of the hemorrhoidal plexus with either an increased arterial inflow, or a decreased venous drainage. If the inflow could be reduced, then the plexus would diminish and the hemorrhoids shrink. ${ }^{5}$ The feeding arteries of the hemorrhoidal plexus are detected by combining a proctoscope with a Doppler transducer, and thus they can be selectively ligated.

In the present study, we evaluated the DGHAL technique in the treatment of symptomatic hemorrhoids.

\section{Patients and methods:}

In the period from January 2007 to December 2008, fifty patients with symptomatic hemorrhoids were treated by DGHAL Figure(1). All patients were subjected to a detailed clinical examination and the degree of hemorrhoids was documented. Barium 
enema and/or colonoscopy were done in selected patients to exclude other pathology. Patients with the following conditions were excluded from the study:

1- Acute thrombosed hemorrhoids.

2- Concomitant anal disease (fissure, fistulas or abscess).

3- Inflammatory bowel disease or other organic colorectal pathology

4- Patients with hematological disorders.

5- Patients on anticoagulant therapy. The procedure was explained thoroughly to every patient and an informed consent was obtained.

Preoperative preparation included clear oral fluids for one day before the procedure and a fleet enema the night of the procedure. Prophylactic antibiotics were not routinely prescribed.



Figure (1): Doppler machine.

\section{Operative technique:}

The patient received general or spinal anaesthesia and was placed in the lithotomy position. The anal region was sterilized and draped. The anal canal was lubricated with electro-conductive gel. The Doppler probe was inserted into the anal canal and the probe was gently rotated to localize hemorrhoidal arteries by hearing good arterial signals. Once the artery was localized, it was ligated with a vicryl stitch mounted on a special needle that suits the procedure Figure(2): vicryl 2/0 mounted on half circumference tapered needle with reinforced needle-thread connection. A figure of eight stitch was placed through the ligation window of the probe and ligated with a knot pusher Figure(3). Obliteration of the vessels was confirmed by the disappearance of any Doppler sounds distal to the suture. The procedure was repeated until all the feeding hemorrhoidal arteries were ligated.

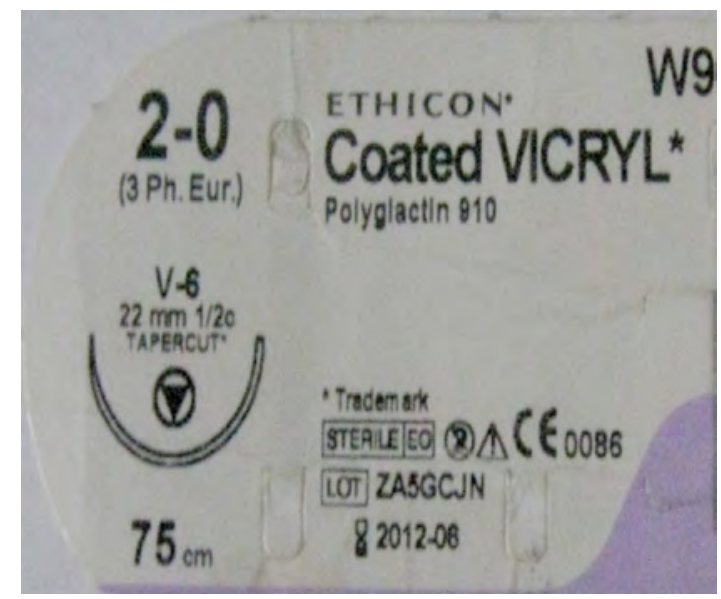

Figure (2): Vicryl 2/0.

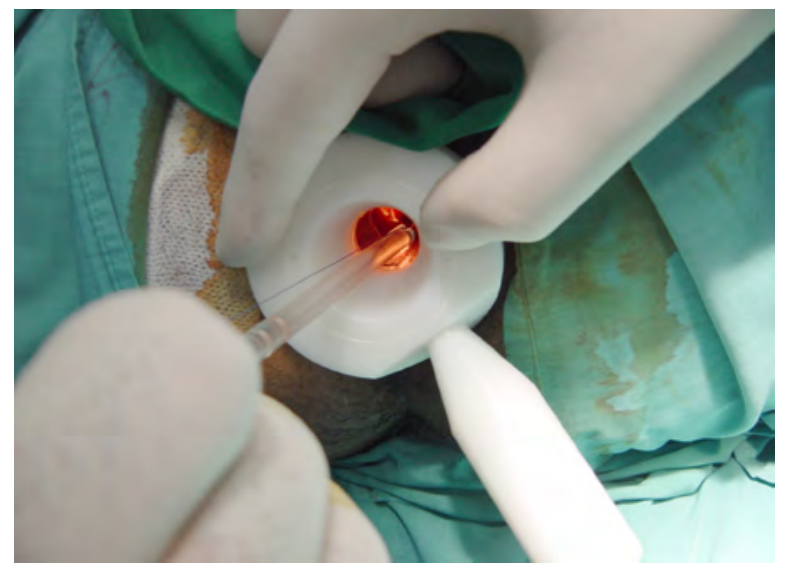

Figure (3): Ligation of the haemorrhoidal artery. 


\section{Post-operative management:}

$>$ Food was allowed after full recovery.

$>$ Pain relief by Declofenac sodium injection (IM) was given to all patients starting 6 hours after operation then by oral analgesic tablets.

> Stool softeners (Agiolax) one sachet /day for one month.

> Daflon for all patients $1000 \mathrm{mg} /$ day orally for a period of 2 weeks

$>$ Hospital discharge was followed under strict criteria:

1- Patients were fully ambulatory.

2- Patients did not complain of bleeding or urinary retention.

$>$ Patients were advised not to have physical strain for another 3 weeks.

$>$ Follow up one week, 1, 3, 6 month and one year.

\section{Measured outcomes:}

The measured outcomes in the present study were:

1- Operative time

2- Postoperative pain is measured by visual analogue scale (VAS) and requirements for analgesics.

3- Postoperative complications

Severe postoperative bleeding was defined as bleeding requiring surgical intervention or hospital readmission.

\section{Results:}

Fifty patients recruited for the present study included 27 males (54\%) and 23 females (46\%). The mean age of patients was $34 \pm 8.6$ years (range 23-64 years). All patients in the present study had symptomatic haemorrhoids and 24 patients $(48 \%)$ had 3rd degree hemorrhoids. It was found that 34 patients $(68 \%)$ of the studied cases had no previous anal operations. The presenting complaint of the studied patients is shown in Table(1).

Table (1): Distribution of the studied cases as regard preoperative symptoms .

\begin{tabular}{|l|c|c|}
\hline Symptoms of Hemorrhoids & No. (50) & \% \\
\hline Pain alone & 20 & $40 \%$ \\
\hline Bleeding + pain & 10 & $20 \%$ \\
\hline Anal swelling & 6 & $12 \%$ \\
\hline Itching + anal swelling & 8 & $16 \%$ \\
\hline Itching & 6 & $12 \%$ \\
\hline
\end{tabular}

Thirty five patients $(70 \%)$ had general anesthesia. The mean number of hemorrhoidal vessels ligated was $4 \pm 0.6$ (range $3-6$ ) vessels and the mean operative time was $32 \pm 3.6$ (range 19 - 45) minutes Table(2).

Table (2): Distribution of the studied cases as regard operative procedures.

\begin{tabular}{|l|c|c|}
\hline Operative procedures & No. & $\%$ \\
\hline Type of anesthesia & & \\
General & 35 & $70 \%$ \\
Spinal & 10 & $20 \%$ \\
Local & 5 & $10 \%$ \\
\hline Vessels ligated (HAL) (mean \pm SD) & $4 \pm 0.6$ & $3-6$ \\
\hline Duration of the operation (minutes) & $32 \pm 3.6$ & $19-45$ \\
\hline
\end{tabular}


All patients received Diclophenac sodium ampule in the early postoperative period then additional analgesics were given according to the patients need. The average need for analgesics requirement from day 1 to day 13 was $225 \pm 35$ to $140.9 \pm 20 \mathrm{mg}$ Declofenac Na Table(3).
The pain score on the first and second day was $6.1 \pm 1.3$ and $5 \pm 1.2$ respectively on a VAS. The following days, the pain was in the form of minimal discomfort which was perceived by patients on a VAS.

Table (3): Distribution of the studied cases as regard VAS and quantity of analgesic consumption.

\begin{tabular}{|c|c|c|}
\hline $\begin{array}{c}\text { Day s after } \\
\text { the operation }\end{array}$ & $\begin{array}{c}\text { VAS } \\
\text { (Mean }+ \text { SD) }\end{array}$ & $\begin{array}{c}\text { Quantity of analgesics required } \\
\text { (diclofenac Na) mg } \\
\text { (Mean } \pm \text { SD) }\end{array}$ \\
\hline 1st day & $6.1 \pm 1.3 \mathrm{~cm}$ & $225 \pm 35 \mathrm{mg}$ \\
\hline Day 2 & $5 \pm 1.2 * \mathrm{~cm}$ & $156 \pm 24 * \mathrm{mg}$ \\
\hline Day 3 & $4.3 \pm 1.9 * * \mathrm{~cm}$ & $140.9 \pm 20 * * \mathrm{mg}$ \\
\hline Day 4 & $3.8 \pm 1.5^{* *} \mathrm{~cm}$ & $136 \pm 25^{* *} \mathrm{mg}$ \\
\hline Day 5 & $3 \pm 1.1 * * \mathrm{~cm}$ & $76.2 \pm 10 * * \mathrm{mg}$ \\
\hline Day 6 & $2.1 \pm 0.6 * * \mathrm{~cm}$ & 0 \\
\hline Day 7 & $2 \pm 0.3 * * \mathrm{~cm}$ & 0 \\
\hline
\end{tabular}

This table shows that VAS together with quantity of analgesics required were decreased markedly from day 1 until day 7 with statistically significant difference in comparison to the baseline (dayl) by paired t-test.

* $P<0.05 S$.

$* * P<0.001 \mathrm{HS}$.

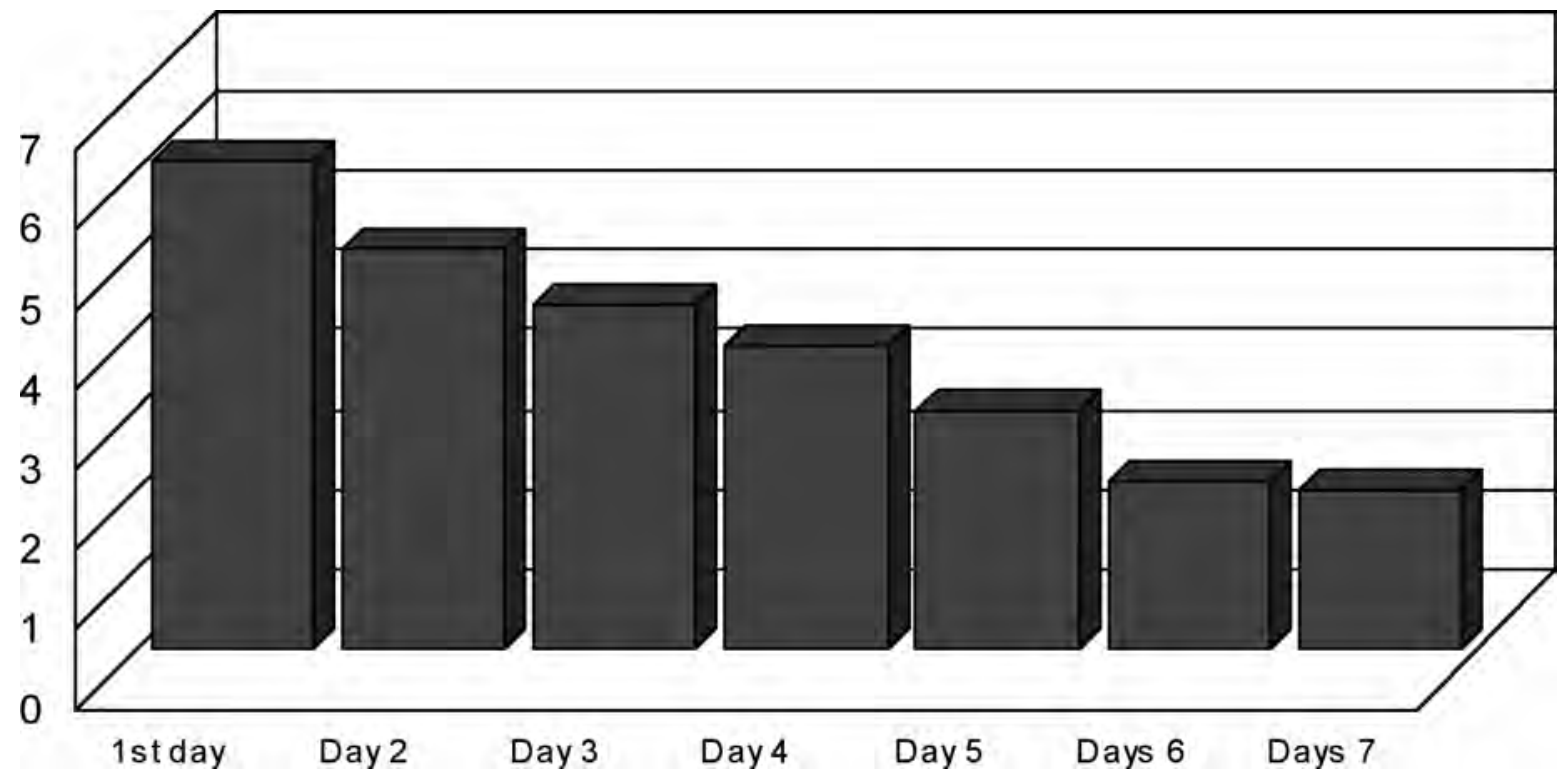

Distribution of the studied cases as regard VAS. 


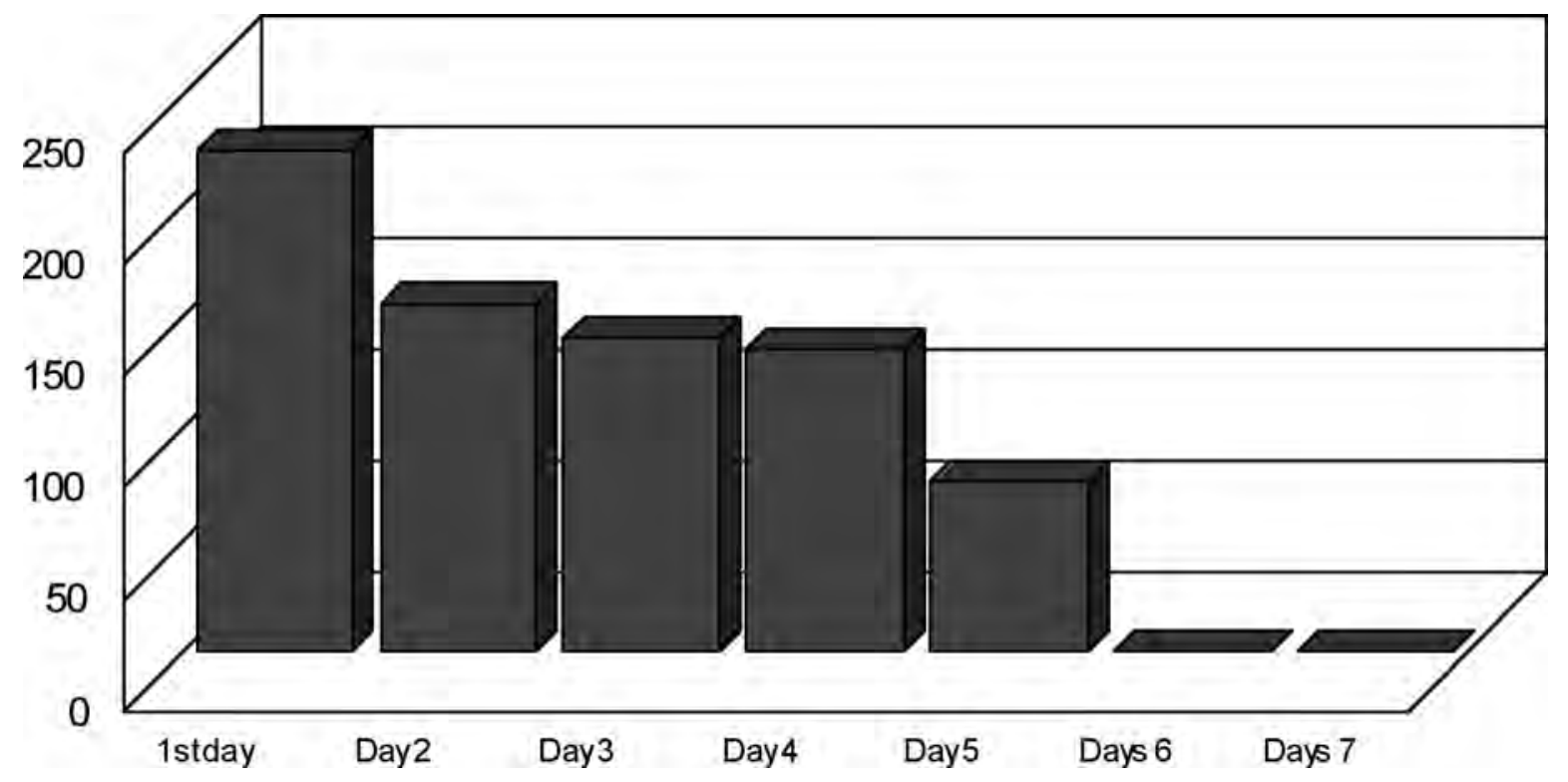

Distribution of the studied cases as regard quantity of analgesic consumption.

Six patients (12\%) developed mild postoperative bleeding. Mild spotting occurred in $12 \%$ of the studied cases, while no cases had severe bleeding, and $6 \%$ of the studied cases had thrombosis in the external pile. Three patients $(6 \%)$ complained of persistent prolapse with long term follow up but pain was improved Table(4).

Table (4): Distribution of the studied cases as regard postoperative complications.

\begin{tabular}{|l|c|c|}
\hline Complications & No. & $\%$ \\
\hline Thrombosis in the external pile & 3 & $6 \%$ \\
\hline Postoperative bleeding & & \\
Mild (spotting) & 6 & $12 \%$ \\
Severe & 0 & 0 \\
\hline Urinary retention & 1 & $2 \%$ \\
\hline Pruritis & 3 & $6 \%$ \\
\hline Prolalpse & 3 & $6 \%$ \\
\hline Local suppuration & 1 & $2 \%$ \\
\hline
\end{tabular}

\section{Discussion:}

Surgical haemorrhoidectomy is considered to be the gold standard in treating haemorrhoidal disease. Much research over the last two decades has concentrated on reducing post haemorrhoidectomy pain resulting from this surgical procedure. ${ }^{9}$

Research has been concentrated on two areas: Modifications of the technique of surgical hemorrhodiectomy and the use of a variety of surgical instruments in the hope of decreasing postoperative pain. ${ }^{10}$

Although many treatment modalities have been described in the literature, no technique has been conclusively demonstrated to be superior. ${ }^{11,12}$

DG-HAL is a relatively new treatment modality based on an accurate localization of hemorrhoidal arteries by Doppler probe, which are individually ligated with figure of eight 
sutures on the level of rectal mucosa without excision or tissue necrosis. It became clear to us that reduction of blood supply into the hemorrhoidal plexus reduces the size of prolapse but the prolapse does not always fully disappear.

The HAL technique results in minimal postoperative pain in comparison with surgical hemorroidectomy and can be done as a day case procedure in outpatient clinic with early return to work and so patients' approval is extremely high.

Patients were examined after one week and then at 1, 3, 6 months thereafter.

In our study, five patients (10\%) complained of pain after the procedure and received analgesics for two weeks. One patient (2\%) developed purulent discharge in the 5 th post operative day and was treated conservatively with antibiotics. Six patients $(12 \%)$ were complaining of mild postoperative bleeding and were treated conservatively. Three patients (6\%) were found to have persistent prolapse but pain disappeared. Three patients $(6 \%)$ complained of pruiritis and received topical steroid cream. One patient (2\%) developed urinary retention postoperatively after receiving spinal anesthesia and was relieved by urinary catheterization.

Complications such as secondary hemorrhage, incontinence, and anal stenosis were not recorded.

Morinaga et al's initial study reported promising results using the HAL on 116 patients. One month post the procedure symptoms of bleeding had stopped in $69 \%$ of the patients, 95\% had pain relief and $78 \%$ had improvement of prolapse symptoms. ${ }^{13}$ These results have been replicated by several other single center studies of large sample sizes. ${ }^{14,15}$ These studies also demonstrated that the technique is well tolerated, is a relatively painless procedure and is able to be performed with reduced anesthetic intervention using sedation and / or local anesthesia. To date there is only 1 published randomized trial comparing conventional hemorrhoidectomy to hemorrhoidal artery ligation. ${ }^{16}$ It reported both techniques to be equally effective in terms of the treatment of symptoms and recurrence rates at 1 year follow up, but found HAL to be less painful and result in reduced length of hospital stay.
These results made us to decide to use DGHAL as the treatment of choice for symptomatic hemorrhoids as it is safe, effective, and low cost with minimal long term complications.

\section{References:}

1- Janssen LWM: Consensus hemorroiden [in Dutch]. Ned Tijdschr Geneeskd 1994; 138: 2106-2109.

2- Goligher J, Duthie H, Nixon H: Hemorrhoids or Piles. Surgery of the anus, rectum and colon. London: Bailliere Tindall (Publisher);5th edn. 1992; p.144-145.

3- Shanmugam V, Thaha MA, Rabindranath KS, Campbell KL, Steele RJC, Loudon MA: Systematic review of randomized trials comparing rubber band ligation with excisional haemorrhoidectomy. Br J Surg 2005; 92: 1481-1487.

4- Senapati A, Nicholls RJ: A randomised trial to compare the results of injection sclerotherapy with a bulk laxative alone in the treatment of bleeding haemorrhoids. Int J Colorectal Dis 1988; 3: 124-126.

5- Bursics A, Morvay K, Kupcsulik P and Flautner L: Comparison of early and 1year follow-up results of conventional hemorrhoidectomy and hemorrhoid artery ligation: A randomized study. Int $J$ Colorectal Dis 2004; 19: 176-180.

6- Longo A: Treatment of haemorrhoids disease by reduction of mucosa and haemorrhoidal prolapsed with cicular suturing device; a new procedure. 6th World Congress Of Endoscopic Surgery (Rome) 1998; p. 777784.

7- Narro JL: Hemorrhoid therapy with Doppler guided hemorrhoidal artery ligation via proctoscope KM-25. A new alternative to hemorrhoidectomy and rubber band ligation? Zentralbl Chir 2004; 129: 208210.

8- Hussein AM: Ligation-anopexy for treatment of advanced hemmoroidal disease. Dis Colon Rectum 2001; 44: 1887-1890.

9- Milligan ETC, Morgan CN Joones LE, et al: Surgical anatomy of the anal canal, and the operative treatment of haemorrhoids. Lancet 1937; 2: 1119-1124.

10-Lacerda-Filho A, Cunha-Melo JR: Outpatient haemorrhoidectomy under local 
anaesthesia. Eur J Surg 1997; 163: 935940.

11-Hosch SB, Knoefel WT, Pichlmeier U, et al: Surgical treatment of piles: Prospective, randomized study of Parks vs. MilliganMorgan hemorrhoidectomy. Dis Colon Rectum 1998; 41: 159-164.

12-Seow-Choen F, Low HC: Prospective randomized study of radical versus four piles haemorrhoidectomy for symptomatic large circumferential prolapsed piles. $\mathrm{Br}$ J Surg 1995; 82: 188-189.

13-Morinaga K, Hasuda K, Ikeda T: A novel therapy for internal hemorrhoids: Ligation of the hemorrhoidal artery with a newly devised instrument (Moricorn) in a conjugation with a Doppler flowmeter. Am J Gastroenteral 1995; 90: 610-613.
14-Scheyer M, Antonietti E, Rollinger G, Mall $\mathrm{H}$, Arnold S: Doppler guided hemorrhoidal artery ligation. Am J Surg 2006; 191: 8993.

15-Lienert M, Ulrich B, Doppler guided ligation of the hemorrhoidal arteries. Report of experiences with 248 patients. Dtsch Med Wochenschr 2004; 129: 947-950.

16-Bursics A, Morvay K, Kepusulik P, Flautner $\mathrm{L}$ : Comparison of early and 1 year follow up results of conventional hemorrhoidectomy and hemorrhoid artery ligation: A randomized study. Int $J$ colorectal Dis 2004; 19: 176-180. 